\title{
Introduction of Multi-particle Büttiker Probes - Bridging the Gap between Drift Diffusion and Quantum Transport
}

\author{
Kuang-Chung Wang, ${ }^{1}$ Roberto Grassi, ${ }^{2}$ Yuanchen Chu, ${ }^{1}$ Shree Hari Sureshbabu, ${ }^{1}$ Junzhe \\ Geng, ${ }^{1}$ Prasad Sarangapani, ${ }^{1}$ Xinchen Guo, ${ }^{1}$ Mark Townsend, ${ }^{2}$ and Tillmann Kubis ${ }^{1,3,4,5}$ \\ ${ }^{1}$ School of Electrical and Computer Engineering, \\ Purdue University, West Lafayette, IN 47906, USA \\ ${ }^{2}$ Silvaco Co., Santa Clara, CA , USA \\ ${ }^{3}$ Network for Computational Nanotechnology, Purdue University, West Lafayette, IN 47906, USA \\ ${ }^{4}$ Purdue Center for Predictive Materials and Devices, \\ Purdue University, West Lafayette, IN 47906, USA \\ ${ }^{5}$ Purdue Institute of Inflammation, Immunology and Infectious Disease, West Lafayette, IN 47906, USA
}

(Dated: January 14, 2020)

\begin{abstract}
State-of-the-art industrial semiconductor device modeling is based on highly efficient DriftDiffusion (DD) models that include some quantum corrections for nanodevices. In contrast, latest academic quantum transport models are based on the non-equilibrium Green's function (NEGF) method that cover all coherent and incoherent quantum effects consistently. Carrier recombination and generation in optoelectronic nanodevices represent an immense numerical challenge when solved within NEGF. In this work, the numerically efficient Büttiker-probe model is expanded to include electron-hole recombination and generation in the NEGF framework. Benchmarks of the new multiple-particle Büttiker probe method against state-of-the-art quantum-corrected DD models show quantitative agreements except in cases of pronounced tunneling and interference effects.
\end{abstract}

\section{INTRODUCTION}

State-of-the-art semiconductor device fabrication techniques allow for device design at the atomistic length scale 1. The performance of nanodevices is equally influenced by coherent quantum mechanical phenomena (such as confinement, tunneling and interference) [2]3 and incoherent scattering of electrons on device imperfections and lattice vibrations 45. The performance of solar cells [67], lasers [8] and light emitting diodes [910] critically depend on the incoherent interaction of electrons with phonons and photons and the interplay between radiative, Auger and Shockley-Read-Hall (SRH) recombination. Carrier generation and recombination affect the off-current and switching characteristics of tunneling field effect transistors 1112. The non-equilibrium Green's function method (NEGF) is among the most general methods to describe coherent and incoherent transport physics [5]. Due to the large numerical load when incoherent scattering is included in the self-consistent Born approximation [5], NEGF is typically applied in the coherent transport limit [13]. This is particularly problematic in nanodevices with pronounced incoherent effects 14 .

There are various algorithms to include incoherent scattering in NEGF. The self-consistent Born approximation can rigorously treat incoherent scattering [5], carrier generation [15] and recombination [16]. However, the self-consistent Born approximation involves several nonlinear and highly dimensional integro-differential equations, which yield high computational load. The multiscale and multi-physics NEGF implementation of Ref. 9 had been designed for modeling light emitting diodes with low numerical load. It requires full charge carrier thermalization in each quantum well. Electron-hole recombination process is limited to the fully thermalized quantum wells as well. The Büttiker-probe model 1719] represents a good compromise between the accuracy of NEGF and the numerical efficiency of heuristic scattering models for devices with incomplete carrier thermalization. In this work, the Büttiker-probes are extended to cover electron-hole recombination and generation in addition to its traditional application space of mobility limiting intraband scattering. Current conservation for intraband and interband scattering is ensured. NEGF predictions with the augmented Büttiker-probes are benchmarked against the drift-diffusion(DD) method of Atlas 20. DD is at the core of industrial technology computer aided design (TCAD) tools for micro-scale devices [2122]. DD is known for its computational efficiency, but it requires to additional correction terms for mimicking coherent quantum effects [23 25]. The NEGF with Büttiker-probe transport predictions of pnjunctions agree quantitatively with DD results of the ON-current density and with results of the density in thermalized device regions. pn junctions that include quantum wells can serve as solar cells 26 27] and photodetectors [28]. Therefore, the new method is also benchmarked against a quantum corrected DD model for carrier recombination and light absorption. Deviations are found in cases with pronounced tunneling and interference effects. It is worth to mention the method is compatible with arbitrary basis representations, ranging from effective mass 29] and k.p [30] to atomistic approaches [3134 . 


\section{METHOD}

The new Büttiker probes are benchmark against stateof-the-art TCAD methods on two devices - a GaN pn diode and a GaN pn diode including an intrinsic InGaN quantum well centered at the p-n interface. GaN electrons and holes are modeled each in effective mass assuming the isotropic masses $m_{e}=0.2 m^{*}$ and $m_{h}=$ $1.25 m^{*} 35$. The position dependent electron and hole recombination and generation [36] rate $\left(R_{R / G}\right)$ depends on the contributions of $\mathrm{SRH}$, radiative recombination, Auger effect and light absorption

$$
\begin{aligned}
& R_{R / G}(x) \\
& =R_{S R H}(x)+R_{\text {radiative }}(x)+R_{\text {Auger }}(x)+R_{G}(x) .
\end{aligned}
$$

The SRH recombination rate is given by Ref. 36.

$$
\begin{aligned}
& R_{S R H}(x) \\
& =\frac{N_{n}(x) N_{p}(x)-n_{\text {intrinsic }}^{2}}{\left(N_{n}(x)+n_{\text {intrinsic }}\right) / A+\left(N_{p}(x)+n_{\text {intrinsic }}\right) / A}
\end{aligned}
$$

with $A=2.6 \times 10^{6} s^{-1}$, the empirical parameter of the inverse recombination lifetime [213738], $n_{\text {intrinsic }}$ the intrinsic carrier density 39 and $N_{n, p}$, the density of electrons $(\mathrm{n})$ and holes $(\mathrm{p})$. We solve the radiative recombination rate by 36 .

$$
R_{\text {radiative }}(x)=B \cdot N_{n}(x) N_{p}(x)
$$

with the empirical parameter 213738 $B=1.48 \times$ $10^{-11} \mathrm{~cm}^{3} \mathrm{~s}^{-1}$. We determine the Auger recombination rate with 36 .

$$
R_{\text {Auger }}(x)=C \cdot\left(N_{n}(x)^{2} N_{p}(x)+N_{p}(x)^{2} N_{n}(x)\right)
$$

with the empirical parameter 213738 $C=1.6 \times$ $10^{-30} \mathrm{~cm}^{6} \mathrm{~s}^{-1}$. Note that we use the same ABC parameters for all NEGF and TCAD results literature. In most calculations, the generation current density $R_{G}(x)$ is set to 0 . Only when explicitly mentioned that illumination is included, the generation current density $R_{G}$ in the well is determined by integrating photon numbers of the solar spectrum of energies larger than the bandgap of $\operatorname{In}_{0.13} \mathrm{Ga}_{0.87} \mathrm{~N}$. Outside the well, $R_{G}(x)$ is assumed to vanish

$$
R_{G}(x)= \begin{cases}R_{G}, & x \in \text { InGaN } \\ 0, & \text { otherwise }\end{cases}
$$

The performance of the two devices is solved in NEGF with the new Büttiker probes. The NEGF results are benchmarked against DD and quantum corrected DD model (SILVACO-ATLAS[20]) results.

For all models, the spatially resolved recombination and generation rate $\left(R_{R / G}(x)\right)$ is multiplied with the elementary charge and integrated along the total device to solve for the total recombination and recombination current density $J_{R / G}$.

$$
J_{R / G}=\int_{0}^{L} R_{R / G}(x) d x .
$$

For each carrier type a current conservation law modified by the recombination and generation is fulfilled

$$
\begin{aligned}
& J_{s, n}+J_{d, n}+J_{R / G}=0 \\
& J_{s, p}+J_{d, p}-J_{R / G}=0 .
\end{aligned}
$$

$J_{s / d, n}\left(J_{s / d, p}\right)$ defines the source/drain current density for electrons (holes). The total measurable current density at the source is given as

$$
J_{\text {total }}=J_{s}=J_{s, n}+J_{s, p} .
$$

An equivalent equation holds for the drain current density.

\section{A. NEGF with Büttiker probes}

Electron and hole properties are solved within the NEGF method [40]. To limit the computational load, the devices are partitioned and Green's functions are solved recursively on the resulting slabs [41]. The retarded Green's function $G^{R}$ is solved by the Dyson equation 40.

$$
G^{R}=\left[E-H-\Sigma_{S}^{R}-\Sigma_{D}^{R}-\Sigma_{B P}^{R}\right]^{-1} .
$$

The lesser Green's function $G^{<}$is given in the Keldysh equation

$$
G^{<}=G^{R}\left(\Sigma_{S}^{<}+\Sigma_{D}^{<}+\Sigma_{B P}^{<}\right) G^{R \dagger} .
$$

All Green's functions and self-energies are matrices in the discretized positions space. Their dependency on the inplane momentum $k_{\|}$and energy $E$ is omitted in Eqs. (9) and 10 for better readability. The source and drain contact self-energies are given by $\Sigma_{S}^{R,<}$ and $\Sigma_{D}^{R,<}$. Scattering of electrons and holes is included with Büttiker probe self-energies $\left(\Sigma_{B P}^{R,<}\right)$ [41 43]. Quantities such as the density of states, the particle density, the state occupancy and the current density can be deduced from the Green's functions as typical for the NEGF method 4044.

The retarded Büttiker probe combines all intraband scattering processes, such as scattering on various phonons, impurities and electron-electron scattering into the empirical scattering parameter $\eta$ [9]. To resemble the Urbach tail 45 46] in GaN, $\eta$ is exponentially decaying into the band gap. For electrons in the conduction band, the retarded Büttiker probe self-energy reads

$$
\begin{aligned}
& \Sigma_{B P, n / p}^{R}\left(x, x^{\prime}, k_{\|}, E\right)=\delta\left(x-x^{\prime}\right) \\
& \times \begin{cases}a \eta_{n}, & \text { if } E \geq E_{c}\left(x, k_{\|}\right) \\
a \eta_{n} \cdot \exp \left(-\frac{E_{c}\left(x, k_{\|}\right)-E}{\lambda}\right), & \text { if } E_{c}\left(x, k_{\|}\right)>E \geq \frac{E_{c}+E_{v}}{2} \\
a \eta_{p} \cdot \exp \left(\frac{E_{v}\left(x, k_{\|}\right)-E}{\lambda}\right), & \text { if } \frac{E_{c}+E_{v}}{2}>E \geq E_{v}\left(x, k_{\|}\right) \\
a \eta_{p}, & \text { if } E_{v}\left(x, k_{\|}\right)>E .\end{cases}
\end{aligned}
$$


In all NEGF calculations, the mesh size a is set to $0.259 \mathrm{~nm}$.

The retarded Büttiker probe for holes in the valence band has the same formula, but with valence band parameters. Similar to Ref. 47, the electron and hole mobility are deduced from the respective resistivity of nor p-doped homogeneous material samples solved with NEGF and Büttiker probes

$$
\rho_{n, p}=\frac{d R_{n, p}^{\Omega}}{d L}=\frac{1}{q\left(\tilde{\mu}_{n, p} N_{n, p}\right)} .
$$

Here, $R^{\Omega}$ refers to the resistance of GaN samples of length $L, R^{\Omega}(L)=V_{s d} / I(L)$. The applied Fermi level difference of source and drain in the mobility calculation is set to $10 \mathrm{meV} . I(L)$ is the length dependent current density for electrons or holes and solved for $L=20 \mathrm{~nm}$ and $L=$ $25 \mathrm{~nm}$. The empirical scattering parameter $\eta_{n}=0.05 \mathrm{eV}$ for electrons and $\eta_{p}=0.06 \mathrm{eV}$ holes are chosen such that the respective NEGF predicted mobility agrees with $\tilde{\mu}_{e}=56.88 \mathrm{~cm}^{2} /(V s)$ and $\tilde{\mu}_{h}=10.0 \mathrm{~cm}^{2} /(\mathrm{Vs})$ (taken from Ref. 48). For completeness, Fig 1 shows the GaN hole and electron mobility as a function of the respective $\eta$. The band tail parameter $\lambda$ is chosen to be $55 \mathrm{meV}$ for electrons according to Ref. 49. The same value is assumed for holes.

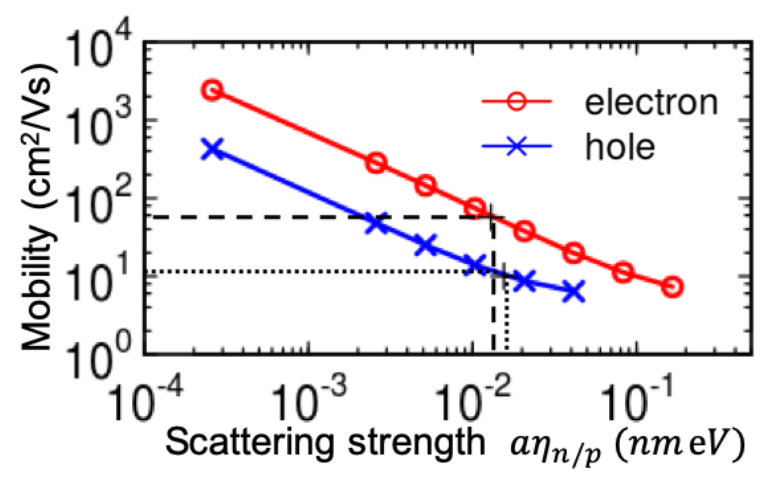

FIG. 1. NEGF predicted mobility of a homogeneous semiconductor with $\mathrm{p}$ and $\mathrm{n}$ doping density of $10^{20} / \mathrm{cm}^{3}$ as a function of the product of $\eta_{n, p}$ of Eq. (11) with the mesh spacing $a$.

The "lesser than" Büttiker probe self-energy $\Sigma_{B P}^{<}$is depending on the Büttiker probe Fermi-levels, $\mu_{n / p}(x)$

$$
\begin{aligned}
& \Sigma_{B P, n / p}^{<}\left(x, x^{\prime}, k_{\|}, E\right)= \\
& \left\{\begin{array}{l}
-F\left(\mu_{n}(x), E\right)\left(\Sigma_{R}\left(x, x^{\prime}, k_{\|}, E\right)-\Sigma_{R}^{\dagger}\left(x, x^{\prime}, k_{\|}, E\right)\right), \\
\text { if } E \geq \frac{E_{c}+E_{v}}{2} \\
-\left(1-F\left(\mu_{p}(x), E\right)\left(\Sigma_{R}\left(x, x^{\prime}, k_{\|}, E\right)-\Sigma_{R}^{\dagger}\left(x, x^{\prime}, k_{\|}, E\right)\right),\right. \\
\text { if } E<\frac{E_{c}+E_{v}}{2} .
\end{array}\right.
\end{aligned}
$$

Here, $F$ is the equilibrium Fermi distribution function. In this work, $\mu_{n}(x)$ and $\mu_{p}(x)$ are solved iteratively to satisfy the overall current conservation

$$
R_{n}(x)=R_{p}(x)=R_{R / G}(x)
$$

$R_{n}$ and $R_{p}$ represent the net electron and hole current of the Büttiker probe at position $x$, respectively. In the state-of-the-art Büttiker probe models, $\mu_{n}(x)$ and $\mu_{p}(x)$ are solved separately which ensures both, electron and hole current conservation individually. Equation (14) agrees with the common Büttiker probe model for the case of $R_{R / G}=0$.

The retarded source and drain self-energies $\Sigma_{S, D}^{R}$ are solved iteratively following Ref. [5051]. To guarantee smooth electron and hole transitions at the device/source and device/drain interfaces, the retarded Büttiker probe self-energy of Eq. (11) is included in the source and drain self-energy calculation 52 53. The Büttiker probe and NEGF equations are iterated with the Poisson equation to achieve charge self-consistency. Piezoelectric and spontaneous polarizations are included as well following Ref. 9 .

\section{B. Drift diffusion models}

The purely semiclassical bulk drift-diffusion(DD) model [54 is applied on the homojunction pn diode with a mesh spacing of $0.1 \mathrm{~nm}$. In case of the pn diode including a quantum well, the DD model is augmented with quantum corrections for the bound states (DD+qwell) in the well region 20. That heterojunction device is discretized with an adaptive real space mesh [20] with average mesh spacing of $0.16 \mathrm{~nm}$. Depending on their energy and location, electrons and holes are separated into two groups, "bound states" and "bulk states". For electronic energies below the barrier potentials ("bound states") at the GaN/InGaN interfaces, the Schrödinger equation is solved assuming the wave functions vanishing at the Schrödinger domain boundaries (i.e. Dirichlet boundary conditions). The solution domain of the Schrödinger equation is exceeding the InGaN quantum well by $10 \mathrm{~nm}$ in both directions to account for the wavefunction penetration into the barriers. Artificial bound states in GaN that would arise from the Dirichlet boundary conditions are avoided by limiting the lower bound of the GaN band edge to the barrier potential at the $\mathrm{GaN} / \mathrm{InGaN}$ interfaces [20. For "bulk states", the DD equations are solved throughout the structure, but the InGaN band edge is shifted to the minimum of the barrier potentials at the unaltered GaN/InGaN interfaces 20/55]. This ensures all "bulk states" do not face quantum well potential confinement.

Charge carriers of the two energy sets ("bound" and "bulk"), are coupled to each other by a capture-escape model 20. Capture-escape rates for electrons and holes respectively are added to the DD continuity equations to allow transitions between "bound" and "bulk" particle groups [20]. Recombination-generation mechanisms are included in the continuity equations as well (Eqs. (2 4) ). Detailed balance is ensured and all Fermi levels uniquely determined by using the same rates for carrier gain/loss in the "bound" and "bulk" groups and for generation and 
recombination, respectively. For charge self-consistency, the sum of the electron and hole "bound" and "bulk" density is iterated with the Poisson equation.

\section{RESULT}

Three application scenarios (a pn-diode, a pn-diode with a quantum well and an illuminated pn-diode with quantum well) are used to benchmark the Büttiker probe model against the semiclassical and quantum corrected semiclassical models. The comparison shows the two methods agree very well in situations without pronounced quantum effects. The quantum corrections of the semiclassical model capture quantum effects but the results still deviate from those of a pure quantum mechanical treatment.

The pn diode is composed of $10 \mathrm{~nm}$ p type and $10 \mathrm{~nm}$ $\mathrm{n}$ type doped GaN with the doping concentration of $10^{20} / \mathrm{cm}^{3}$ in each region. Both regions are periodic in the transverse directions. The pn diode with the quantum well differs from the pn diode by a $3.0 \mathrm{~nm}$ thick intrinsic $\mathrm{In}_{0.13} \mathrm{Ga}_{0.87} \mathrm{~N}$ quantum well layer. If not explicitly mentioned otherwise, the temperature is assumed to be $350 \mathrm{~K}$.

\section{A. GaN pn junction}

Figure 2(a) shows the position resolved band edge of the GaN pn diode solved in DD and NEGF. Both results agree quantitatively. The charge distributions of the two methods are depicted in Fig. 2(b). The majority carrier in each region shows agreement between the two models while the minority charge from NEGF is around $10^{3}$ and $10^{5}$ times higher in the depletion region for electrons and hole, respectively. The minority carriers enter the oppositely doped area due to tunneling as illustrated by the contour plot of the energy resolved carrier density of NEGF in Fig. 2(a). Note, the tunneling is longer ranged for conduction band states with their lighter effective mass than for the states in the valence band. DD-based models do not capture this tunneling effect and the state-of-the-art quantum corrections do not apply to "bulk states". The energy resolved density also illustrates Urbach tails with the additional density at energies below (above) the conduction (valence) band edge. It is worth to mention, decreasing $\lambda$ to $5 \mathrm{meV}$ in Eq. (11) reduced the Urbach tail and decreases the OFF-current by $10 \%$ while the ON-current changes only marginally.

Differences in the density entail deviations of the recombination current density of the two models (see Eqs. (2)-(4)). This is illustrated in Fig. 3 (a) which shows the various contributions to the position resolved recombination rate of DD and NEGF with Büttiker probes for the situation in Fig. 2(b). For higher applied bias, the effective barrier between $\mathrm{n}$ - and p-doped region reduces. Minority carrier tunneling becomes less relevant

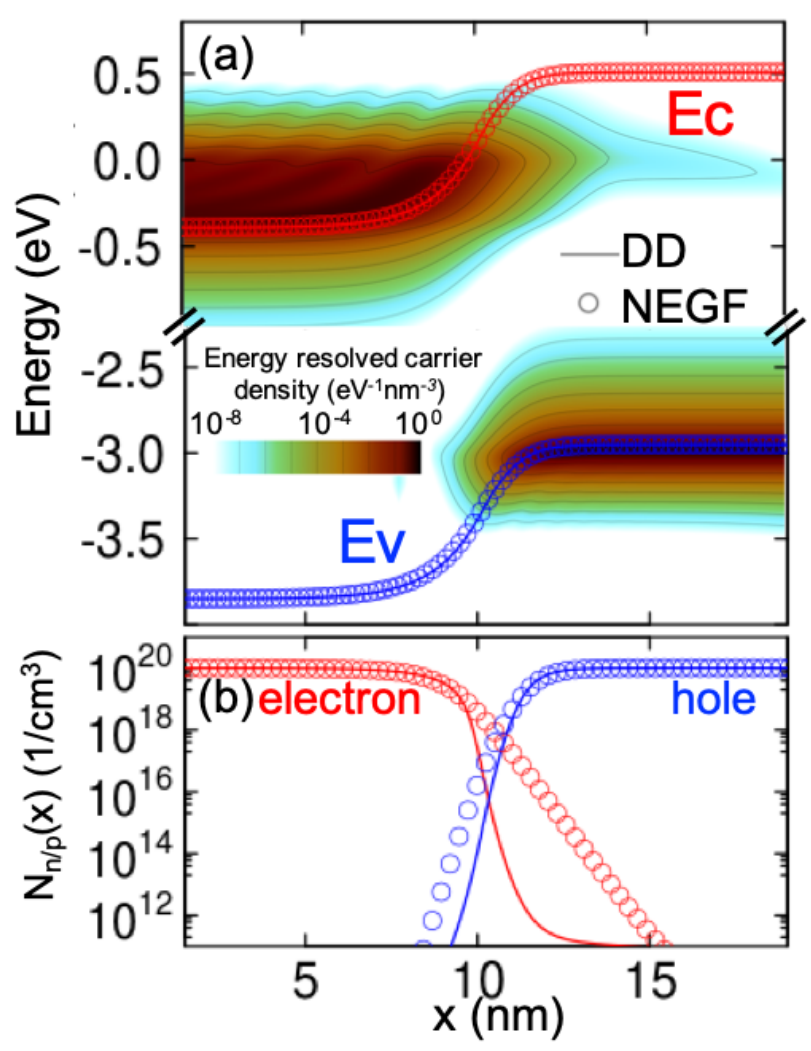

FIG. 2. The GaN pn diode described in the main text with a voltage of $V_{s d}=3.0 \mathrm{~V}$ applied. (a) Conduction and valence band profiles solved in DD (lines) and NEGF with Büttiker probes (symbols) along with contour plots of the energy resolved carrier densities at vanishing in-plane momentum. (b) Position resolved electron and hole densities solved in DD (lines) and NEGF with Büttiker probes (symbols).

compared to the thermionic emission current [56. In consequence, the densities of DD and NEGF with Büttiker probes at a higher voltages agree better and so do the recombination rate contributions in Fig. 3(b).

The comparison of the total current density (Eq. 88) predicted with DD and NEGF with Büttiker probes follows the same trend as can be seen in Fig, 4. Low voltages show pronounced deviations, whereas voltages above about $3.2 \mathrm{~V}$ yield quantitive agreement in the total current density. The recombination current density deviates until about $3.5 \mathrm{~V}$, but its relative contribution is insignificant for voltages above $3.2 \mathrm{~V}$. Note that the slope of the IV-curve below $V_{s d}=3.4 \mathrm{~V}$ differs significantly between the two models due to the different treatment of tunneling. 


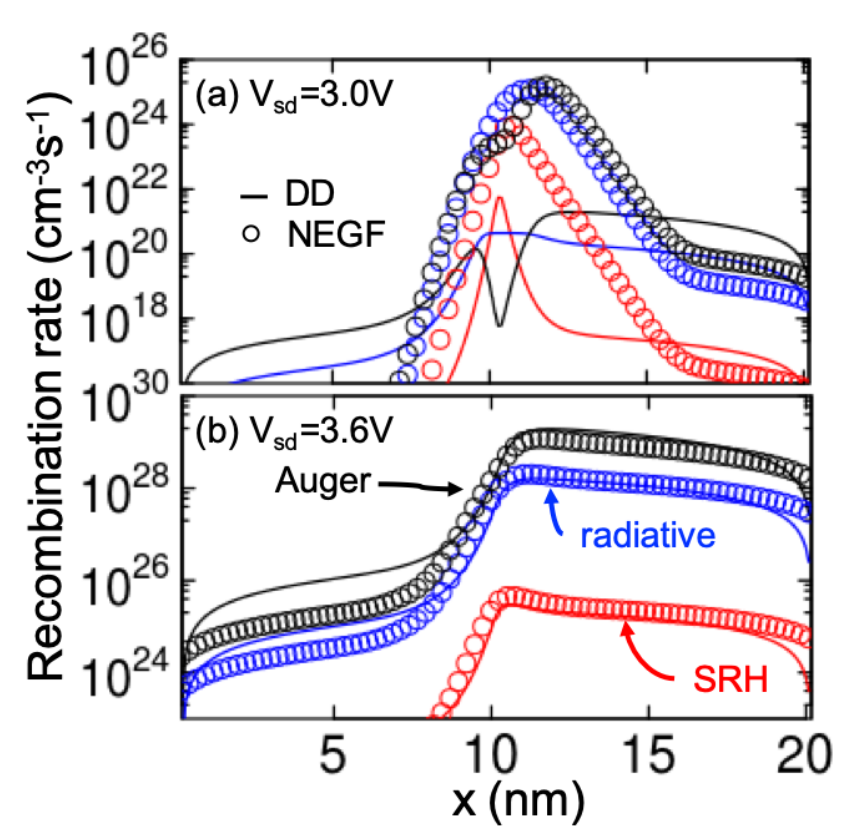

FIG. 3. Spatially resolved recombination rates $\left(R_{S R H}(x)\right.$, $R_{\text {radiative }}(x)$ and $\left.R_{\text {Auger }}(x)\right)$ for the pn diode of Fig. 2 solved in NEGF with Büttiker probes (symbols) and DD (lines) for applied voltages of $\mathrm{V}_{s d}=3.0 \mathrm{~V}$ (a) and $3.6 \mathrm{~V}(\mathrm{~b})$.

\section{B. InGaN quantum well embedded in GaN pn junction}

When an $\operatorname{In}_{0.13} \mathrm{Ga}_{0.87} \mathrm{~N}$ layer is added to the center of the GaN pn junction, a quantum well in the conduction and valence band forms (see Fig. 5(a)). Unaltered DD density results follow the band profiles [54]. Consequently, the unaltered DD calculations yield maxima in the carrier density close to the $\operatorname{In}_{0.13} \mathrm{Ga}_{0.87} \mathrm{~N} / \mathrm{GaN}$ interfaces (see Fig. 5(b)). In contrast, quantum corrected calculations cover quantum confinement effects with wavefunction maxima of "bound states" closer to the quantum well center (see dashed lines for conduction $\Psi_{n, q w e l l}$ and valence $\Psi_{p, q w e l l}$ band "bound states" in Fig. 5(a)).

Calculations of NEGF with Büttiker probes do not distinguish between confined and continuum states but allow for smooth transitions between them. This is illustrated with the contour plot of the energy and position resolved density of states (DOS) in Fig. 5(a).

Confined carriers in the quantum well extend into the continuum of states. Continuum states are also modified by the interference effects at the quantum well boundaries. Higher order quantum well states with energies well beyond the barrier heights are still visible in the DOS continuum (yellow lines in Fig. 5(a)). These effects are missed in the quantum corrected DD model (DD+qwell) that separates "bound" and "continuum" spectra. In detail, the conduction and valence band ground state energies predicted in NEGF are approximately $0.13 \mathrm{eV}$ higher

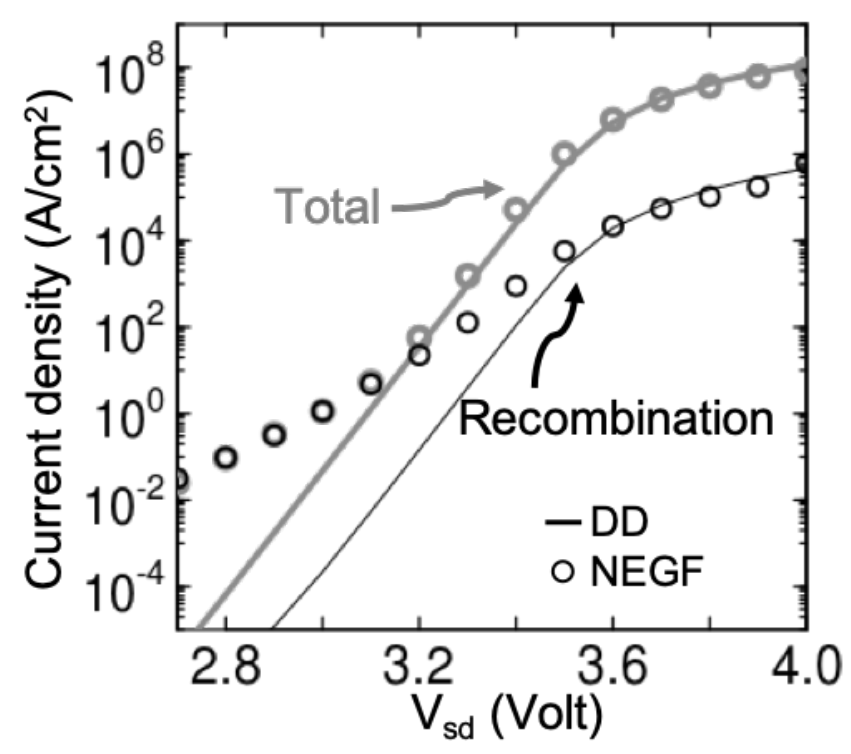

FIG. 4. Current-voltage characteristics of the pn diode of Fig. 2 predicted by NEGF with Büttiker probes (symbols) and DD (lines). The total current density (gray) contains the contributions from the recombination current density (black).

than in DD+qwell. In consequence, the local density of the three models differ (see Fig. 5(b)).

For an applied voltage of $\mathrm{V}_{s d}=4.0 \mathrm{~V}$ the quantum well ground states of conduction and valence bands are well confined (see Fig. 6. In this case, a distinction of "bound" and "continuum" states as done in DD+qwell is obvious. However, the energy resolved density in Fig. 6 for energies above the barrier potential (i.e. "continuum" states) still shows significant interference due to the potential change at the quantum well. The interference pattern of the electron (hole) density are best visible in the n-doped (p-doped) region. These quantum effects add resistance to the Büttiker probe scattering. Since the Büttiker probe scattering strength was tuned to match the mobility assumed in the DD calculations, the total current density of the device in Fig. 6 is predicted lower in NEGF than DD. This is illustrated in Fig. 7 which shows the total ON current is about two times smaller in NEGF than DD. Since the density of the confined state in the quantum well of NEGF calculations is smaller than in DD (see Fig. 5b), the recombination current density of NEGF is smaller than in DD, too (see black lines and symbols Fig. 7).

\section{Illuminated InGaN quantum well embedded in GaN pn junction}

When various carrier generation rates due to solar light absorption are included in the calculations of the InGaN quantum well system of Figs. (5)-(7), the predicted current density is linearly shifted to negative values (see 


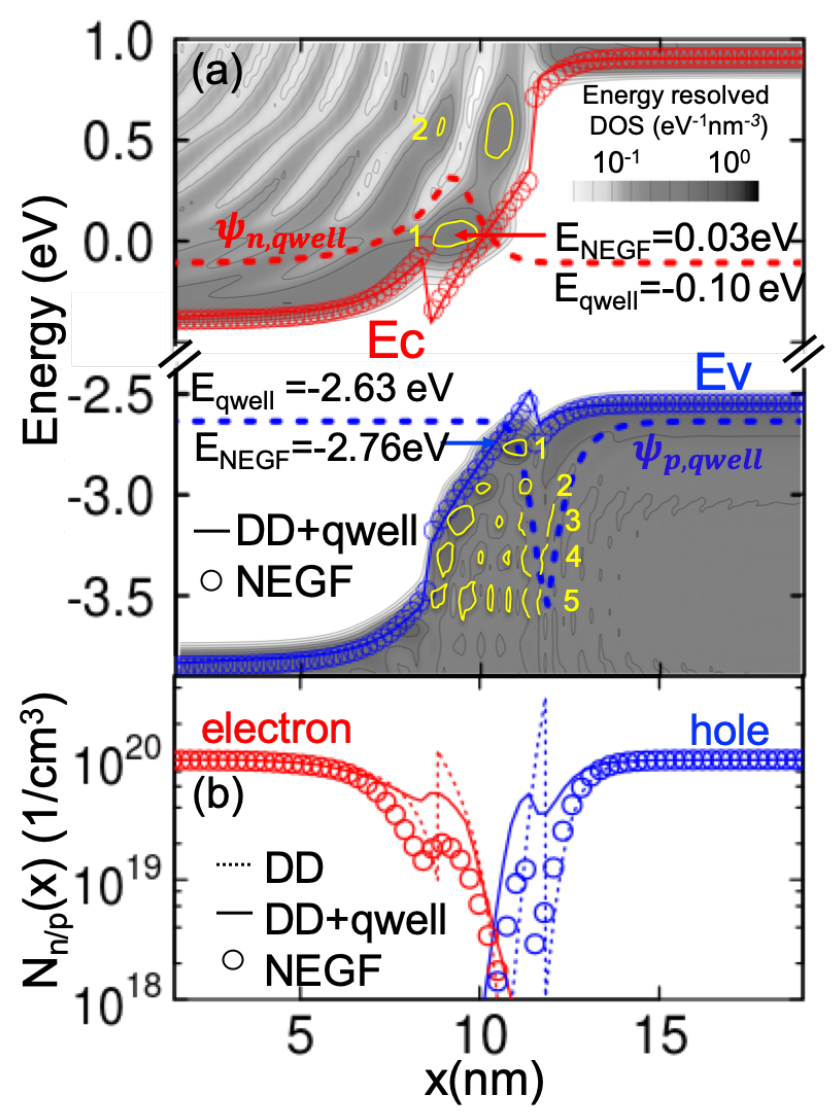

FIG. 5. The GaN pn diode with an InGaN quantum well described in the main text for an applied voltage of $V_{s d}=2.6 \mathrm{~V}$. (a) Conduction and valence band profiles solved in DD+qwell (lines) and NEGF with Büttiker probes (symbols) along with a contour plot of the energy resolved density of states at vanishing in-plane momentum. The dashed lines show the squared absolute value of the "bound" quantum well wave functions in the DD+qwell model. For reasons discussed in the main text, the ground state energies of DD+qwell and NEGF differ, and NEGF covers quasi-bound states in addition (indicated with yellow lines and labeled with numbers). (b) Position resolved electron and hole densities solved in DD+qwell (lines) and NEGF with Büttiker probes (symbols).

Fig. 8) as common for solar cell operations [57. The open circuit voltage for DD calculations at $350 \mathrm{~K}$ temperature is smaller than in the NEGF case. This is a result of the different quantum well densities and recombination rates discussed already in Figs. 5(b) and7. With a lower temperature of $100 \mathrm{~K}$, the ground state energy of the quantum well turns out to be larger than the confinement potential and the DD model does not find any "bound states". Then, the quantum well density of DD calculations is lower than in NEGF, and same holds for the recombination current density. Therefore, at $100 \mathrm{~K}$, a higher open circuit voltage is observed in DD than in NEGF.

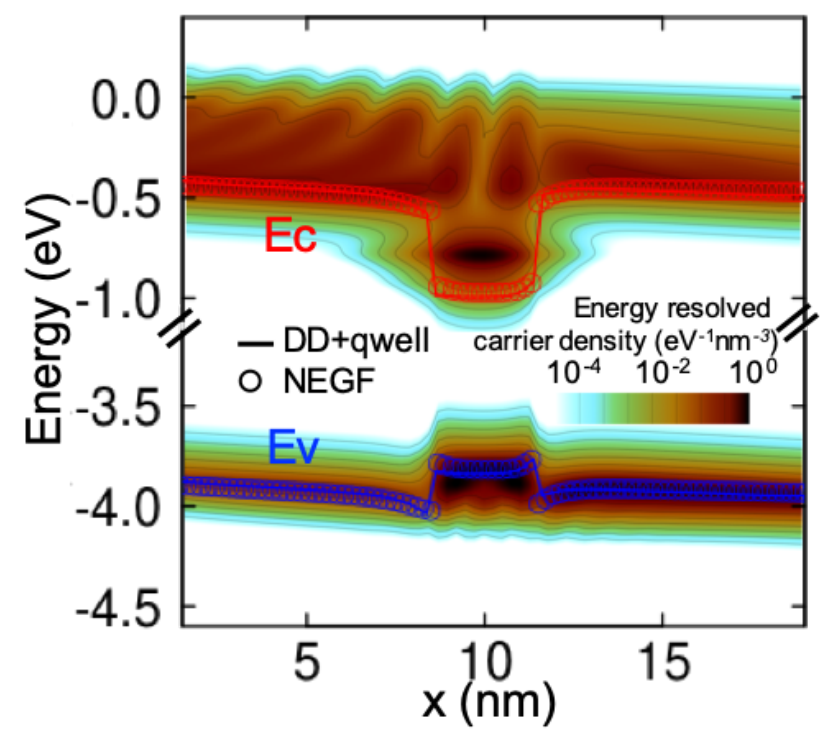

FIG. 6. The GaN pn diode with an InGaN quantum well of Fig. 5 with a voltage of $V_{s d}=4.0 \mathrm{~V}$ applied. Conduction and valence band profiles solved in DD+qwell (lines) and NEGF with Büttiker probes (symbols) are shown along with a contour plot of the energy resolved density of states at vanishing in-plane momentum solved in NEGF with Büttiker probes.

\section{CONCLUSION}

This work augmented the Büttiker probe based scattering model in the nonequilibrium Green's function framework to efficiently model electron-hole recombination and generation processes. Electrons and holes are modeled as separate particles that observe particle continuity equations with explicit particle creation and destruction rates. The combined system of holes and electrons conserves the current. These Büttiker probes are applied on transport of electrons and holes in GaN pnjunctions with and without an embedded InGaN quantum well. The results are benchmarked against DriftDiffusion and quantum-corrected Drift-Diffusion calculations of Silvaco's TCAD tool Atlas [20. The two methods agree quantitatively except for situations with pronounced carrier tunneling and interference. In particular, interference effects in the band continuum can create quasi-bound states that impact the optoelectronic device performance. In consequence and depending on the detailed device geometry, temperature and applied bias the open-circuit voltage of quantum well pn-junctions is underestimated or overestimated in state-of-art TCAD simulations. 


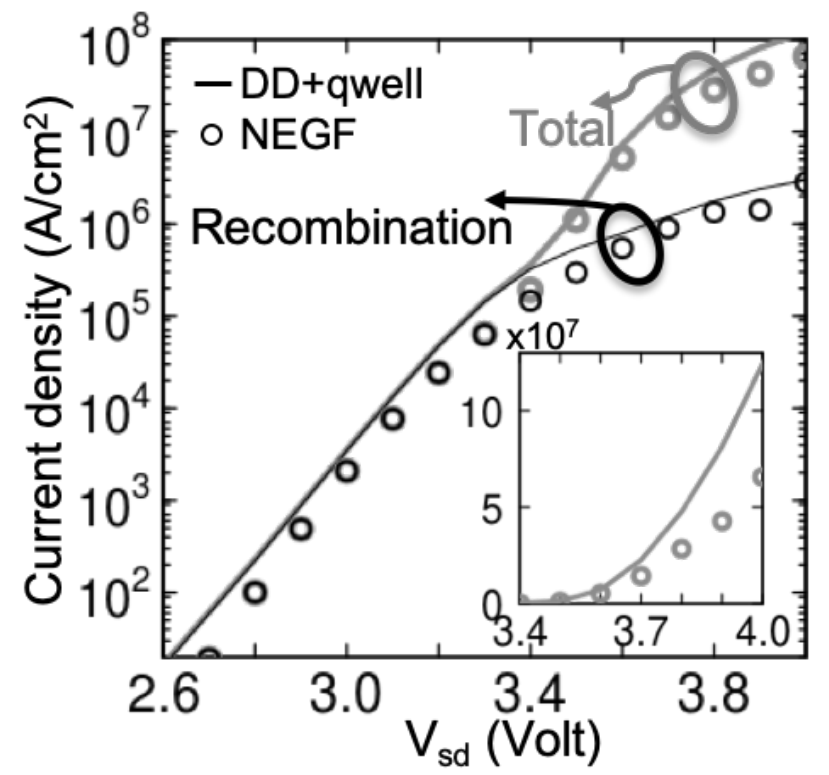

FIG. 7. Current-voltage characteristics of the GaN pn diode with a InGaN quantum well of Fig. 5 predicted by NEGF with Büttiker probes (symbols) and DD+qwell (lines). The total current density (gray) contains the contributions from the recombination current density (black). The inset illustrates a difference of approximately $2 \mathrm{x}$ in the ON-current predicted with NEGF and DD.

\section{ACKNOWLEDGMENT}

We acknowledge the Rosen Center for Advanced Computing at Purdue University for the use of their computing resources and technical support. The authors acknowledge the Texas Advanced Computing Center (TACC) at the University of Texas at Austin for providing high-performance computing resources.
[1] C.-s. Pang, C.-y. Chen, T. Ameen, S. Zhang, H. Ilatikhameneh, R. Rahman, G. Klimeck, and Z. Chen, WSe 2 Homojunction Devices : Electrostatically Configurable as Diodes, MOSFETs, and Tunnel FETs for Reconfigurable Computing, 1902770, 1 (2019).

[2] P. Krogstrup, H. I. Jørgensen, M. Heiss, O. Demichel, J. V. Holm, M. Aagesen, J. Nygard, and A. Fontcuberta I Morral, Single-nanowire solar cells beyond the ShockleyQueisser limit, Nature Photonics 7, 306 (2013).

[3] H. Ilatikhameneh, T. A. Ameen, C. Chen, G. Klimeck, and R. Rahman, Sensitivity Challenge of Steep Transistors, IEEE Transactions on Electron Devices (2018), 10.1109/TED.2018.2808040.

[4] M. Luisier, Atomistic modeling of anharmonic phononphonon scattering in nanowires, Physical Review B Condensed Matter and Materials Physics 86, 1 (2012).

[5] J. Charles, P. Sarangapani, R. Golizadeh-Mojarad, R. Andrawis, D. Lemus, X. Guo, D. Mejia, J. E. Fonseca, M. Povolotskyi, T. Kubis, and G. Klimeck, Incoherent transport in NEMO5: realistic and efficient scattering on phonons, Journal of Computational Electronics 15, 1123 (2016)

[6] M. Burgelman, P. Nollet, and S. Degrave, Modelling polycrystalline semiconductor solar cells, Thin Solid Films 361, 527 (2000).

[7] K.-c. Wang and Y.-r. Wu, Transition Rate in the InGaN Quantum Dot Intermediate-Band Solar Cell, , 822 (2011).
[8] C. Jirauschek and T. Kubis, Modeling techniques for quantum cascade lasers, Applied Physics Reviews 1 (2014), 10.1063/1.4863665

[9] J. Geng, P. Sarangapani, K. C. Wang, E. Nelson, B. Browne, C. Wordelman, J. Charles, Y. Chu, T. Kubis, and G. Klimeck, Quantitative Multi-Scale, Multi-Physics Quantum Transport Modeling of GaN-Based Light Emitting Diodes, Physica Status Solidi (A) Applications and Materials Science 215, 1 (2018)

[10 $]$ S. Pimputkar, J. S. Speck, S. P. Denbaars, and S. Nakamura, Prospects for LED lighting, Nature Photonics 3, 180 (2009)

[11] P. Ghedini, D. Agopian, M. Dalla, V. Martino, S. Gomes, J. Antonio, R. Rooyackers, D. Leonelli, and C. Claeys, Solid-State Electronics Temperature impact on the tunnel fet off-state current components, Solid State Electronics 78, 141 (2012).

[12] Q. Smets, A. S. Verhulst, E. Simoen, D. Gundlach, C. Richter, N. Collaert, and M. M. Heyns, Calibration of Bulk Trap-Assisted Tunneling and Shockley-Read-Hall Currents and Impact on InGaAs Tunnel-FETs, IEEE Transactions on Electron Devices 64, 3622 (2017)

[13] a. Svizhenko, M. P. Anantram, T. R. Govindan, B. Biegel, and R. Venugopal, Two-dimensional quantum mechanical modeling of nanotransistors, Journal of Applied Physics 91, 2343 (2002).

[14] G. Klimeck, R. Lake, R. C. Bowen, W. R. Frensley, and T. S. Moise, Quantum device simulation with a general- 


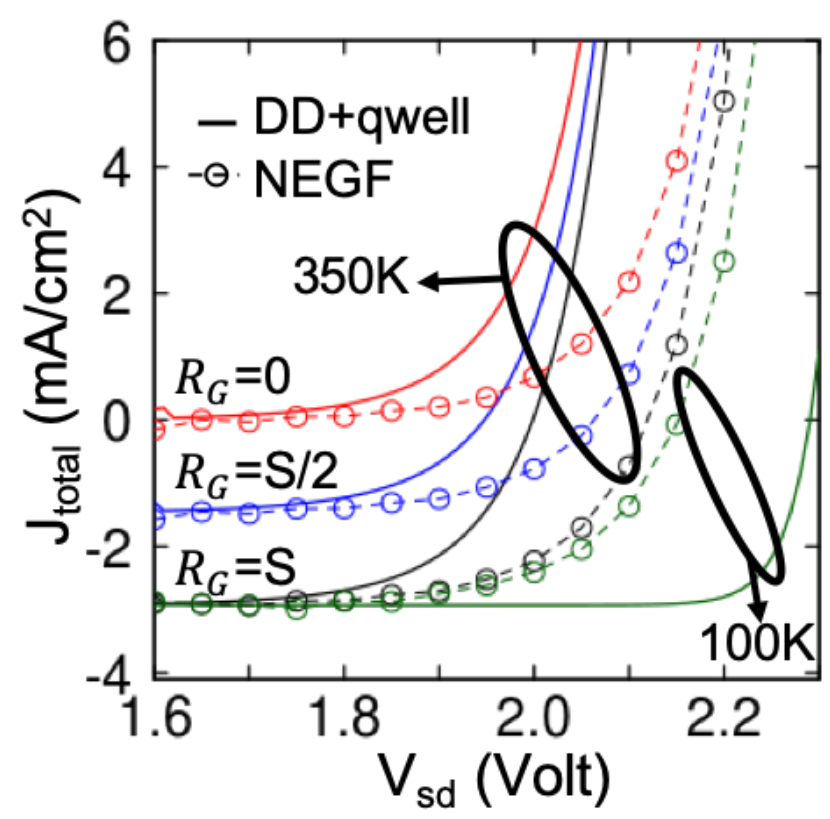

FIG. 8. Current-voltage characteristics of the GaN pn diode with a InGaN quantum well of Fig. 5 predicted by NEGF with Büttiker probes (symbols) and DD+qwell (lines) with a finite electron-hole generation rate of $\mathrm{S}=6.4 \times 10^{22} \mathrm{~cm}^{-3} \mathrm{~s}^{-1}$ in the quantum well. The different quantum mechanical treatment of the bound and quasi-bound states of the two methods leads to differences in the open-circuit voltage at $350 \mathrm{~K}$ and $100 \mathrm{~K}$ as detailed in the main text.

ized tunneling formula, Applied Physics Letters 67, 2539 (1995)

[15] U. Aeberhard, Quantum-kinetic perspective on photovoltaic device operation in nanostructure-based solar cells, Journal of Materials Research 33, 373 (2018).

[16] U. Aeberhard, Nonequilibrium Green's function picture of nonradiative recombination of the Shockley-Read-Hall type, Physical Review B 99, 125302 (2019).

[17] R. Venugopal, M. Paulsson, S. Goasguen, S. Datta, and M. S. Lundstrom, A simple quantum mechanical treatment of scattering in nanoscale transistors, Journal of Applied Physics 93, 5613 (2003)

[18] P. Greck, S. Birner, B. Huber, and P. Vogl, Efficient method for the calculation of dissipative quantum transport in quantum cascade lasers, Optics Express 23, 6587 (2015)

[19] A. Shedbalkar and B. Witzigmann, Non equilibrium Greens function quantum transport for green multiquantum well nitride light emitting diodes, Optical and Quantum Electronics 50, 1 (2018)

[20] Atlas User Manual, Silvaco, Inc., 2811 Mission College Blvd, 6th FL, Santa Clara, CA 95054 (2019)

[21] M. H. Kim, M. F. Schubert, Q. Dai, J. K. Kim, E. F. Schubert, J. Piprek, and Y. Park, Origin of efficiency droop in GaN-based light-emitting diodes, Applied Physics Letters 91, 1 (2007)

[22] Y.-R. Wu, R. Shivaraman, K.-C. Wang, and J. S. Speck, Analyzing the physical properties of In GaN multiple quantum well light emitting diodes from nano scale structure, Applied Physics Letters 101, 083505 (2012).

[23] S. Bandyopadhyay, M. E. Klausmeier-Brown, C. M. Maziar, S. Datta, and M. S. Lundstrom, Rigorous Technique To Couple Monte Carlo and Drift-Diffusion Models for Computationally Efficient Device Simulation. IEEE Transactions on Electron Devices ED-34, 393 (1987).

[24] F. Bufler, A. Schenk, and W. Fichtner, Monte Carlo, Hydrodynamic and Drift-Diffusion Simulation of Scaled Double-Gate MOSFETs, Journal of Computational Electronics 2, 81 (2004).

[25] P. Procel, P. Löper, F. Crupi, C. Ballif, and A. Ingenito, Numerical simulations of hole carrier selective contacts in p-type c-Si solar cells, Solar Energy Materials and Solar Cells 200, 109937 (2019)

[26] J. R. Lang, N. G. Young, R. M. Farrell, Y. R. Wu, and J. S. Speck, Carrier escape mechanism dependence on barrier thickness and temperature in InGaN quantum well solar cells, Applied Physics Letters 101 (2012), $10.1063 / 1.4765068$

[27] J. Wu, W. Walukiewicz, K. M. Yu, W. Shan, J. W. Ager, E. E. Haller, H. Lu, W. J. Schaff, W. K. Metzger, and S. Kurtz, Superior radiation resistance of In1-xGaxN alloys: Full-solar-spectrum photovoltaic material system, Journal of Applied Physics (2003), 10.1063/1.1618353.

[28] S. K. Jain, N. Aggarwal, S. Krishna, R. Kumar, S. Husale, V. Gupta, and G. Gupta, GaN-UV photodetector integrated with asymmetric metal semiconductor metal structure for enhanced responsivity, Journal of Materials Science: Materials in Electronics (2018), 10.1007/s10854-018-8917-3.

[29] J. Wang, E. Polizzi, and M. Lundstrom, A threedimensional quantum simulation of silicon nanowire transistors with the effective-mass approximation, Journal of Applied Physics 96, 2192 (2004)

[30] J. Z. Huang, K. Wang, W. R. Frensley, and G. Klimeck, Finite difference schemes for $k \cdot p$ models: A comparative study, 18th International Workshop on Computational Electronics, IWCE 2015 , 5 (2015)

[31] K. C. Wang, T. K. Stanev, D. Valencia, J. Charles, A. Henning, V. K. Sangwan, A. Lahiri, D. Mejia, P. Sarangapani, M. Povolotskyi, A. Afzalian, J. Maassen, G. Klimeck, M. C. Hersam, L. J. Lauhon, N. P. Stern, and T. Kubis, Control of interlayer physics in $2 \mathrm{H}$ transition metal dichalcogenides, Journal of Applied Physics 122 (2017), 10.1063/1.5005958

[32] D. Valencia, E. Wilson, Z. Jiang, G. A. Valencia-Zapata, K. C. Wang, G. Klimeck, and M. Povolotskyi, GrainBoundary Resistance in Copper Interconnects: From an Atomistic Model to a Neural Network, Physical Review Applied 9, 44005 (2018).

[33] F. Zahid, M. Paulsson, E. Polizzi, A. W. Ghosh, L. Siddiqui, and S. Datta, A self-consistent transport model for molecular conduction based on extended Hückel theory with full three-dimensional electrostatics A self-consistent transport model for molecular conduction based on extended Hückel theory with full three-dimensional el, [ 064707 (2012), 10.1063/1.1961289.

[34] C. Y. Chen, T. A. Ameen, H. Ilatikhameneh, R. Rahman, G. Klimeck, and J. Appenzeller, Channel Thickness Optimization for Ultrathin and 2-D Chemically Doped TFETs, IEEE Transactions on Electron Devices 65, 4614 (2018)

[35] S. K. Pugh, D. J. Dugdale, S. Brand, and R. A. Abram, Electronic structure calculations on nitride semi- 
conductors, Semiconductor Science and Technology 14, 23 (1999)

[36] J. Piprek, Efficiency droop in nitride-based light-emitting diodes, Physica Status Solidi (A) Applications and Materials Science 207, 2217 (2010)

[37] A. Dmitriev and A. Oruzheinikov, The rate of radiative recombination in the nitride semiconductors and alloys, 86 (1999).

[38] F. Olivier, A. Daami, C. Licitra, and F. Templier, Shockley-Read-Hall and Auger non-radiative recombination in GaN based LEDs: A size effect study, Applied Physics Letters (2017), 10.1063/1.4993741.

[39] F. Schäffl, Properties of advanced semiconductor materials : GaN, AlN, InN, BN, SiC, SiGe, John Wiley Sons Inc New York (2001), citeulike-article-id:6144117

[40] S. Datta, Nanoscale device modeling: the Green's function method, Superlattices and Microstructures 28, 253 (2000)

[41] S. Sadasivam, N. Ye, J. P. Feser, J. Charles, K. Miao, T. Kubis, and T. S. Fisher, Thermal transport across metal silicide-silicon interfaces: First-principles calculations and Green's function transport simulations, Physical Review B 95, 1 (2017)

[42] M. Bttiker and M. Buettiker, Role of quantum coherence in series resistors, Physical Review B 33, 3020 (1986)

[43] M. P. Anantram, M. S. Lundstrom, and D. E. Nikonov, Modeling of nanoscale devices, Proceedings of the IEEE 96, 1511 (2008)

[44] T. Kubis and P. Vogl, Assessment of approximations in nonequilibrium Green's function theory, Physical Review B - Condensed Matter and Materials Physics (2011), 10.1103/PhysRevB.83.195304.

[45] Franz Urbach, The Long-Wavelength Edge of Photographic Sensitivity and of the Electronic Absorption of Solids, Physical Review 92, 1324 (1953).

[46] P. Sarangapani, Y. Chu, K. C. Wang, D. Valencia, J. Charles, and T. Kubis, Nonequilibrium Green's function method: Transport and band tail predictions in transition metal dichalcogenides, International Conference on Simulation of Semiconductor Processes and Devices, SISPAD 2018-Septe, 38 (2018)

[47] . Szabó, R. Rhyner, and M. Luisier, Ab initio simulation of single- and few-layer MoS2 transistors: Effect of electron-phonon scattering, Physical Review B - Con- densed Matter and Materials Physics 92, 1 (2015)

[48] T. T. Mnatsakanov, M. E. Levinshtein, L. I. Pomortseva, S. N. Yurkov, G. S. Simin, and M. A. Khan, Carrier mobility model for GaN, Solid-State Electronics 47, 111 (2003)

[49] P. Sarangapani, Y. Chu, J. Charles, and T. Kubis, Nonequilibrium Green's function predictions of band tails and band gap narrowing in III- $V$ semiconductors and nanodevices, (2019)

[50] M. P. L. Sancho, J. M. L. Sancho, and J. Rubio, Quick iterative scheme for the calculation of transfer matrices: application to Mo (100), Journal of Physics F: Metal Physics 14, 1205 (1984)

[51] M. P. L. Sancho, J. M. L. Sancho, J. M. L. Sancho, J. Rubio, H. Search, C. Journals, A. Contact, M. Iopscience, and I. P. Address, Highly convergent schemes for the calculation of bulk and surface Green functions, Journal of Physics F: Metal Physics 15, 851 (1985)

[52] T. Kubis, C. Yeh, P. Vogl, A. Benz, G. Fasching, and C. Deutsch, Theory of nonequilibrium quantum transport and energy dissipation in terahertz quantum cascade lasers, Physical Review B - Condensed Matter and Materials Physics (2009), 10.1103/PhysRevB.79.195323

[53] K. Miao, S. Sadasivam, J. Charles, G. Klimeck, T. S. Fisher, and T. Kubis, Bü ttiker probes for dissipative phonon quantum transport in semiconductor nanostructures, 113107, 1 (2016).

[54] C. de Falco, E. Gatti, A. L. Lacaita, and R. Sacco, Quantum-corrected drift-diffusion models for transport in semiconductor devices, Journal of Computational Physics (2005), 10.1016/j.jcp.2004.10.029.

[55] C.-Y. Chen and Y.-R. Wu, Studying the short channel effect in the scaling of the AlGaN/GaN nanowire transistors, Journal of Applied Physics 113, 214501 (2013)

[56] A. Latreche, Combined thermionic emission and tunneling mechanisms for the analysis of the leakage current for Ga2O3 Schottky barrier diodes, SN Applied Sciences 1, 1 (2019).

[57] D. M. Tobnaghi, R. Madatov, and P. Farhadi, Investigation of light intensity and temperature dependency of solar cells electric parameters, Electric Power Engineering \& Contol Systems 2013 (2013). 